\title{
High Speed OFDM-CDMA Optical Access Network
}

\author{
X. GUO ${ }^{1, *}$, Q. WANG ${ }^{1,3}$, L. ZHOU ${ }^{2}$, L. FANG ${ }^{2}$, A. WONFOR ${ }^{1}$,
}

R. V. PENTY ${ }^{1}$, I. H. WHITE ${ }^{1}$

${ }^{1}$ Centre for Photonic Systems, Electrical Engineering Division, Department of Engineering, University of Cambridge

9 J. J. Thomson Avenue, Cambridge, CB3 OFA, UK

${ }^{2}$ Huawei Technologies, Bantian, Longgang District, Shenzhen 518129, China

${ }^{3}$ Department of Electronic Engineering, Tsinghua University, Beijing 100084, China

*Corresponding author:xg218@cam.ac.uk

Received XX Month XXXX; revised XX Month, XXXX; accepted XX Month XXXX; posted XX Month XXXX (Doc. ID XXXXX); published XX Month XXXX

We demonstrate the feasibility of a $16 \times 3.75 \mathrm{~Gb} / \mathrm{s}(60$ $\mathrm{Gb} / \mathrm{s}$ aggregate) Orthogonal frequency division multiplexing-Code division multiple access passive optical network (OFDM-CDMA PON) for next-generation access applications. $3.75 \mathrm{~Gb} / \mathrm{s}$ PON channel transmission over $25 \mathrm{~km}$ SMF shows $0.1 \mathrm{~dB}$ dispersion and $0.9 \mathrm{~dB}$ crosstalk penalties. Advantages of the system include high capacity, enhanced spectral efficiency, coding gain, networking functions such as increased security and single wavelength operation.

\section{(C) 2015 Optical Society of America}

OCIS codes: (060.4250) Networks; (060.4510) Optical communications; (060.4080) Modulation.

http://dx.doi.org/10.1364/OL.99.099999

The continuing expansion of bandwidth demand for optical access networks is growing exponentially due to the population of bandwidth-hungry services and applications, such as high-definition television (HDTV) and 3-D video telephony, online gaming and cloud computing in recent years which demand optical transmission systems with higher spectral efficiency and higher channel data rates. Optical broadband access networks have emerged to address these challenges by supporting scalable and flexible bandwidths enabling multiple services. Next Generation Passive Optical Networks (NG-
PONs) are forecast to increase the global capacity of broadband access to well beyond $40 \mathrm{~Gb} / \mathrm{s}$ downlink and $10 \mathrm{~Gb} / \mathrm{s}$ uplink levels [1] to meet this need.

However, the computational requirements involved in electronic dispersion compensation for PONs using traditional modulation become very complex and power hungry as the data rate increases. OFDM based PONs are now regarded as promising solutions for future broadband access networks owing to their properties like the high transmission capacity and spectral efficiency, large chromatic and polarization mode dispersion tolerance, and flexible and dynamic bandwidth allocation. In practice, OFDM involves a parallel modulation implementation based on the Fast Fourier Transform (FFT), which enables modulation format variation on each low bandwidth OFDM subcarrier that can adapt to frequency dependent channel quality. This also makes possible simple single-tap equalization, significantly reducing the required bandwidth, complexity and cost of the electronics in the Optical Network Unit (ONU) side [2] [3]. Various OFDM PON configurations have been investigated recently. Time division multiplexing OFDM PONs (TDM-OFDM PONs) [4] which enable efficient and dynamic bandwidth allocation but have difficulties at higher transmission speeds and burst synchronization. They also suffer from low security and high interference between different ONUs and the upstream traffic needs a different wavelength assignment owing to the problem of beat noise. Wavelength division multiplexing OFDM PONs (WDM-OFDM PONs) [5] however can support very high data rates thanks to multiple wavelengths, but are relatively expensive and complex owing to the use of arrayed waveguide gratings and

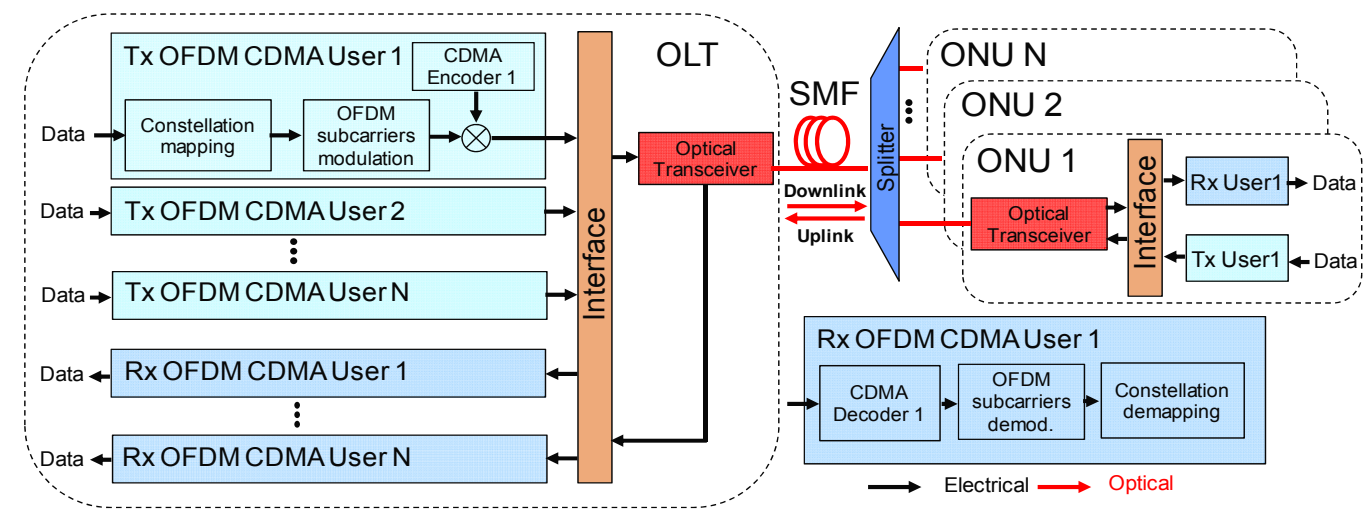

Fig. 1. The proposed architecture of OFDM-CDMA PON. 
multiple high speed OFDM transceivers. They also do not allow flexible and dynamic resource allocation at different ONUs. CDMA technologies have many attractive features such as single wavelength operation, high power budget margins, a secure physical layer, low interference between users and low optical beat noise between different channels [6]. If the OFDM based transmitters and receivers are encoded and decoded with orthogonal codes, they can benefit from dense subcarrier spacing, ease of equalization, multiple access and crosstalk cancellation. Hence the combination of OFDM and CDMA has been shown to be a potential important evolution route for future low cost and high speed NG-PONs [7-8]. Building on this concept, we implement a system using an OFDM modulation scheme which then encounters CDMA encoding/decoding separately for each user for high aggregate data rates. In addition the feasibility of a higher number of users is demonstrated.

Fig. 1 shows the proposed OFDM-CDMA PON architecture. For the downlink direction, the optical line terminal (OLT) generates unique encoded OFDM data streams with orthogonal codes for different users and these are simultaneously transmitted to the ONUs. Each user has a corresponding unique CDMA modem which decodes its own data while the interference between multiple users can be eliminated through code auto- and cross-correlation process in decoding. The decoded data is then demodulated by an OFDM modem. A similar process can also be employed in the uplink to enable bidirectional transmission. This architecture provides several benefits including simple implementation, improved spectral efficiency and security. In particular, it is possible to use a single standard source and detector at the central office to support all users as they share a single wavelength which allows optimization of existing infrastructure and simplifies network management. The correlation properties of the decoding process in a CDMA system also have the potential to improve receiver sensitivity and increase power budgets due to coding gain. This may allow the CDMA signal to be transmitted over longer distances than required in access networks without amplification, thus making possible the integration of access systems with metropolitan area networks (MANs).

Walsh-Hadamard sequences are commonly used for CDMA networks due to their good auto- and cross-correlation properties [6]. For this OFDM-CDMA PON demonstration, a family of WalshHadamard sequences with 16 chips is used. The Walsh-Hadamard codes are defined by the Hadamard matrices of dimension $2^{j}$ for $j \in N_{a}$ ( $N_{a}$ is positive integer) given by the recursive formula below:

$$
\begin{aligned}
H\left(2^{j}\right) & =\left[\begin{array}{cc}
H\left(2^{j-1}\right) & H\left(2^{j-1}\right) \\
H\left(2^{j-1}\right) & -H\left(2^{j-1}\right)
\end{array}\right] \\
& =H(2) \otimes H\left(2^{j-1}\right)
\end{aligned}
$$

for $2 \leq j \leq N_{a}$, where $\otimes$ denotes the Kronecker product. The lowest order of Hadamard matrix of 2 forms the base matrix:

$$
H\left(2^{1}\right)=\left[\begin{array}{cc}
1 & 1 \\
1 & -1
\end{array}\right]
$$

For CDMA encoding and decoding process, different codes (rows of the Walsh-Hadamard matrix) are assigned to different users, and single $l_{\text {th }}$ code from the Walsh-Hadamard matrix is expressed as follows:

$$
\mathbf{c}^{l}=\left[\begin{array}{lll}
c_{0}^{l} & \ldots & c_{M-1}^{l}
\end{array}\right], l=1, \ldots, M
$$

where $M$ denotes the length of the code.

Digital OFDM symbols can be expressed as the output of the inverse FFT (IFFT) with the complex number:

$$
\mathbf{x}=\left[x_{0} x_{1} x_{2} \ldots x_{N-1}\right]^{T}
$$

$$
x_{n}=\frac{1}{\sqrt{N}} \sum_{k=1}^{N} X_{k} \exp \left(\frac{j 2 \pi n k}{N}\right), n=0,1, \ldots, N-1
$$

where $N$ is the size of IFFT.

Different from [7][8], the signals are mapped into all the OFDM subcarriers and then the OFDM symbols in each user are spread and encoded using unique Walsh-Hadamard code rather than the reverse order. Now the $n_{\text {th }}$ CDMA encoded OFDM symbol can be written as:

$$
y_{q}=c_{\bmod (q, M)}^{l} x_{\lfloor q / M\rfloor}, q=0,1,2, \ldots M N-1 .
$$

Mod 0 and \lfloor\rfloor denote the modulus after division and floor process in mathematics. Different users can be transmitted simultaneously and finally in the decoding process, the corresponding Walsh-Hadamard code is used to distinguish each other via auto- and cross-correlation properties.

In the OFDM-CDMA PON experiments, the PRBS bits are mapped to Quadrature Amplitude Modulation (QAM) symbols and then input onto the OFDM subcarriers via a 512-size IFFT. The subcarriers are arranged so that the 2 subcarriers closest to the optical carrier are omitted. The remaining 510 subcarriers have Hermitian symmetry so that the IFFT output is real-valued for intensity modulation-direct detection (IM-DD), though at the cost of lower spectral efficiency. A cyclic prefix (CP) of $3 \%$ is added in front of each OFDM symbol to mitigate the inter-symbol interference (ISI). The complete signal sequence comprises 340 OFDM frames (covering all the subcarriers) in which 40 frames are the training pilots for the estimation of channel subcarriers and phase equalization coefficients. 256 pseudo-noise (PN) bits are inserted in front of the OFDM symbols for automatic system timing synchronization utilizing their strong cross-correlation properties. Each user is encoded with different 16-bit WalshHadamard codes so that ideally 16 users can be transmitted simultaneously. Four typical 16-bit Walsh-Hadamard codes covering different spreading bandwidths are used in this proof-of-principle demonstration.

Code 3: $\{+1+1-1-1+1+1-1-1+1+1-1-1+1+1-1-1\}$

Code $5:\{+1+1+1+1-1-1-1-1+1+1+1+1-1-1-1-1\}$

Code 6: $\{+1-1+1-1-1+1-1+1+1-1+1-1-1+1-1+1\}$

Code $9:\{+1+1+1+1+1+1+1+1-1-1-1-1-1-1-1-1\}$

The parameters for this OFDM-CDMA PON experiments are summarized in Table 1.

\section{Table 1. OFDM-CDMA PON Experimental Parameters}

OFDM-CDMA PON parameters

\begin{tabular}{ll}
\hline Number of bits & 179520 \\
FFT size & 512 \\
Subcarrier bandwidth & $39 \mathrm{MHz}$ \\
Single user data rate & $3.75 \mathrm{~Gb} / \mathrm{s} / 2.5 \mathrm{~Gb} / \mathrm{s}$ \\
Modulation & $64 \mathrm{QAM} / 16 \mathrm{QAM}$ \\
Subcarriers & $2-256$ \\
OFDM frames & 300 \\
Training frames & 40 \\
Synchronization & $256-$-bit PN sequence \\
CP ratio & $3 \%$ \\
CDMA & 16 -bit Walsh-Hadamard codes \\
\hline
\end{tabular}



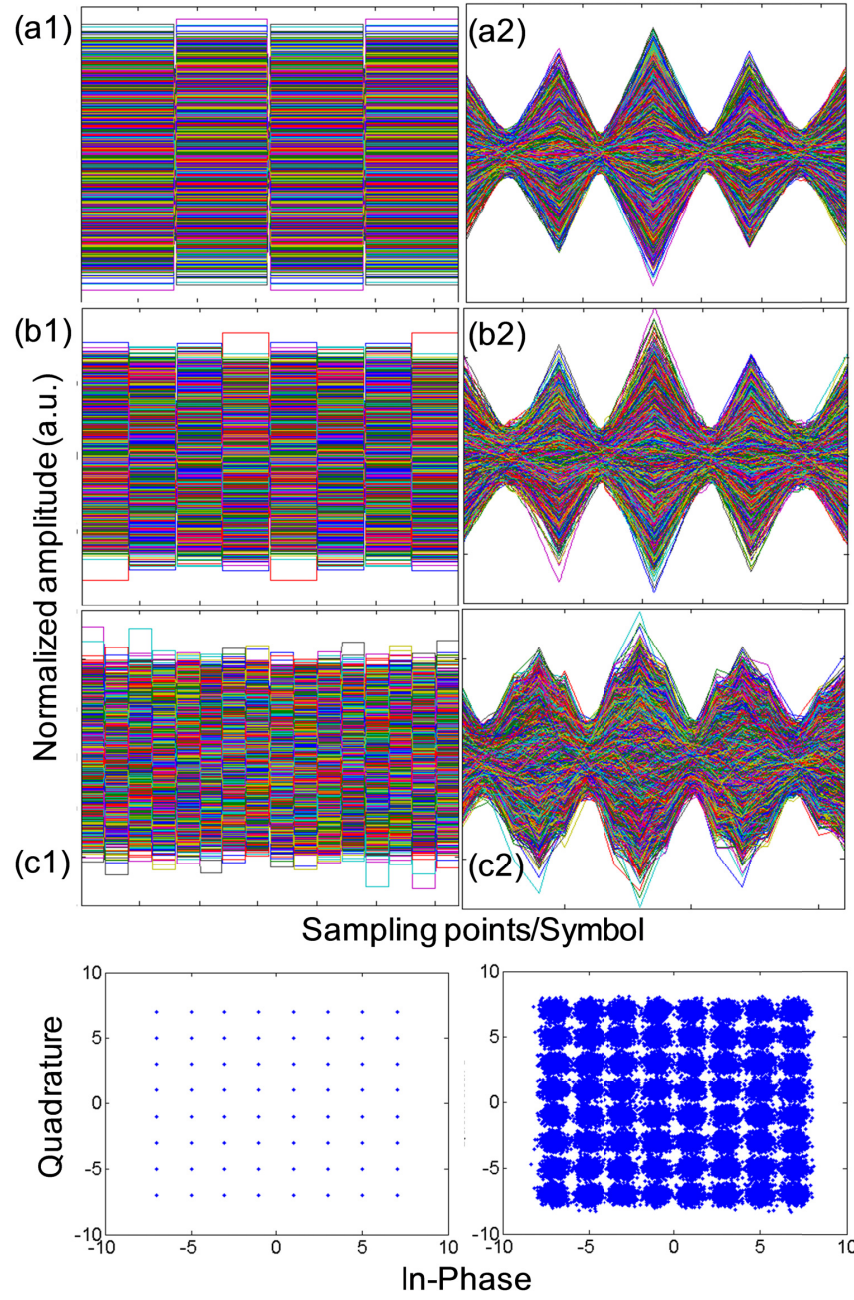

Fig. 2 (a1-c1) Simulation of 64 QAM OFDM symbols with single user (encoded with code 5), combined two users (encoded with code 3 and code 5) and combined four users (encoded with code 3, code 5, code 6 and code 9). (a2-c2) Simulation of decoded signals using code 5R in single, two and four user-systems respectively at BER of $1 \times 10^{-3}$. (d1d2) Simulation of 64 QAM constellation of decoded signals using code $5 \mathrm{R}$ in four-user system at no error and BER of $1 \times 10^{-3}$ two scenarios.

Simulations are carried out first with the same channel data rate $(3.75 \mathrm{~Gb} / \mathrm{s})$ and CDMA parameters as used in the experiments in order to validate the feasibility of the proposed OFDM CDMA PON architecture,. In order to perform correlation, the decoder is set with the reverse of the transmitted chip code. For example, if Code 5 is used in the transmitter, the decoder must be configured using Code 5R: $\{-1$ $1-1-1+1+1+1+1-1-1-1-1+1+1+1+1\}$. The transmitted code then causes the filter to output a high energy signal at the desired sampling point. Fig. 2 (a1-c1) show the simulation results of the single users encoded with code 5, combined two-user system (code 3 and code 5) and combined four-user system (code 3, code 5, code 6 and code 9) respectively but in the absence of noise for the sake of clarity. Fig.2 (a2c2) show the signal in these three scenarios decoded using code $5 \mathrm{R}$ respectively with bit-error-rate (BER) measured to be $1 \times 10^{-3}$ which is the FEC limit. Fig.3 (d1-d2) show the 64 QAM constellation of decoded signal using code $5 \mathrm{R}$ in four-user system at no error and BER of $1 \times 10^{-3}$ two scenarios.

Fig. 3 shows the OFDM-CDMA PON experimental configuration to validate the proposed architecture. We only demonstrate downstream transmission in this paper due to equipment limitations. The OFDMCDMA data is generated offline by Matlab and output by an Arbitrary
Waveform Generator (AWG) with a fixed sampling rate of $20 \mathrm{GS} / \mathrm{s}$ throughout all the experiments. The data modulates a $1550 \mathrm{~nm}$ DFB laser via a $20 \mathrm{GHz} \mathrm{LiNbO}_{3}$ Mach-Zehnder modulator (MZM). The DFB laser is an ILX Lightwave 79800D C-band module with full power of 10 $\mathrm{dBm}$ and wavelength/attenuation accuracy of $\pm 0.05 \mathrm{~nm} / \pm 0.1 \mathrm{~dB}$. The resulting optical signal is then transmitted through $25 \mathrm{~km}$ of SMF, EDFA and an optical bandpass (BF) filter, before being detected by a 20 GHz receiver (-15 dBm sensitivity @10 Gb/s NRZ ). The resulting electrical signal is then amplified by a low noise RF amplifier and sampled by a $100 \mathrm{GS} / \mathrm{s}$ real-time scope for DSP offline processing.

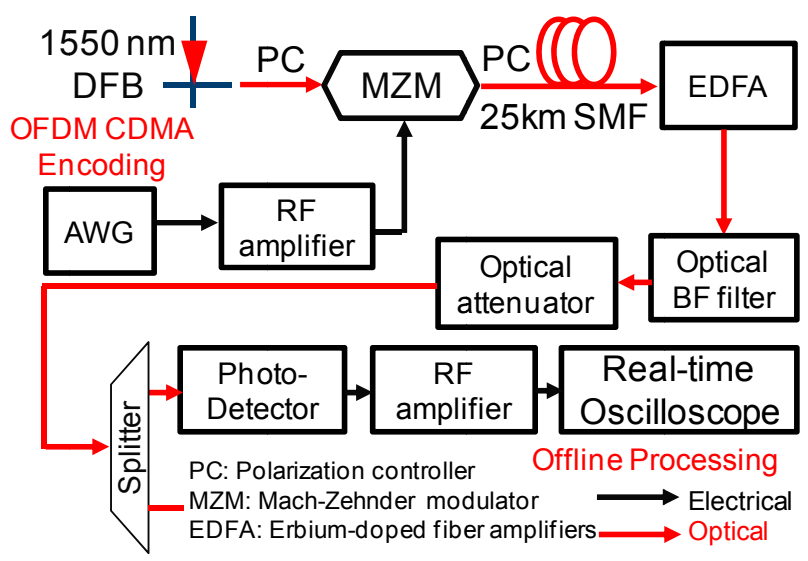

Fig. 3. OFDM-CDMA PON experimental setup (downlink).

The channel frequency response of different OFDM subcarriers is estimated first for the case where the power of all the subcarriers modulated by BPSK is equal. The SNR is estimated at an optical power of $-10 \mathrm{dBm}$ and is shown in Fig. 4 as a function of subcarrier number. It can be seen that the subcarriers occupying high frequencies suffer from larger losses, which reflect the whole system frequency response. Adaptive bit and power loading is applied to improve the spectral efficiency.

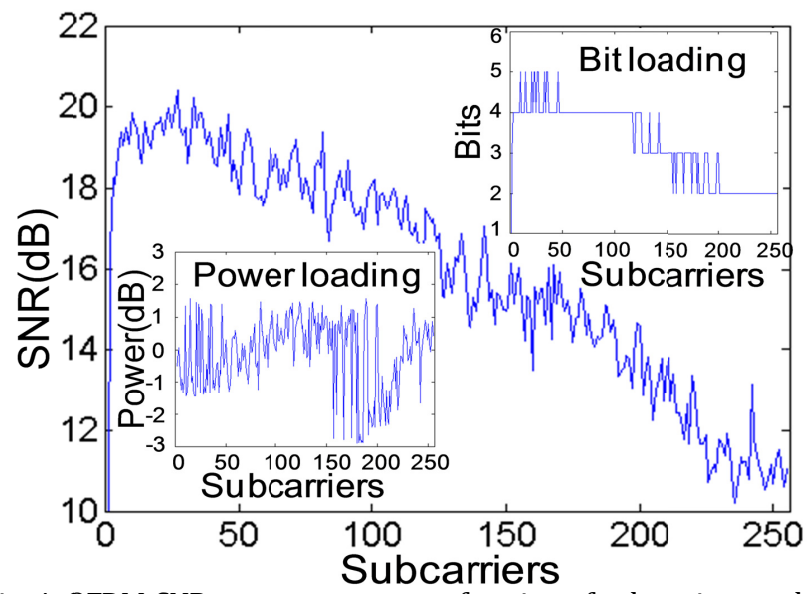

Fig. 4. OFDM SNR measurement as a function of subcarrier number with bit and power loading shown in the insets.

Fig. 5 shows the BER measurements for pure OFDM signals for back-to-back (B2B) operation and after $25 \mathrm{~km}$ of SMF. The spectral efficiency is measured to be $1.62 \mathrm{bit} / \mathrm{s} / \mathrm{Hz}$ and the total data rate is 32.4 $\mathrm{Gb} / \mathrm{s}$. There is a negligible $0.1 \mathrm{~dB}$ measured power penalty, which indicates good dispersion tolerance. Fig. 5 also depicts the BER curves of 64 QAM OFDM CDMA one and two user cases (code 5 and 9) after $25 \mathrm{~km}$ of SMF. In order to simplify the experiment, all the subcarriers the adaptive power/bit loading is not applied. It is clearly shown that 
there is about a $2.8 \mathrm{~dB}$ improvement when CDMA coding is applied and this will increase further if a lower order modulation format is used. Despite there being a $3 \mathrm{~dB}$ power splitting loss for two users, there is nearly no measured crosstalk penalty for two simultaneous users. The BER performance is better than the FEC limit in each case. Fig. 5 also shows the constellation maps of received 64 QAM signal at BERs of $1.1 \times 10^{-3}$ and $2 \times 10^{-4}$ respectively for the two-user system. The spectral efficiency is $3 \mathrm{bit} / \mathrm{s} / \mathrm{Hz}$ and a single user data rate can achieve $3.75 \mathrm{~Gb} / \mathrm{s}$, showing the potential for a record high aggregate data rate of $60 \mathrm{~Gb} / \mathrm{s}$.

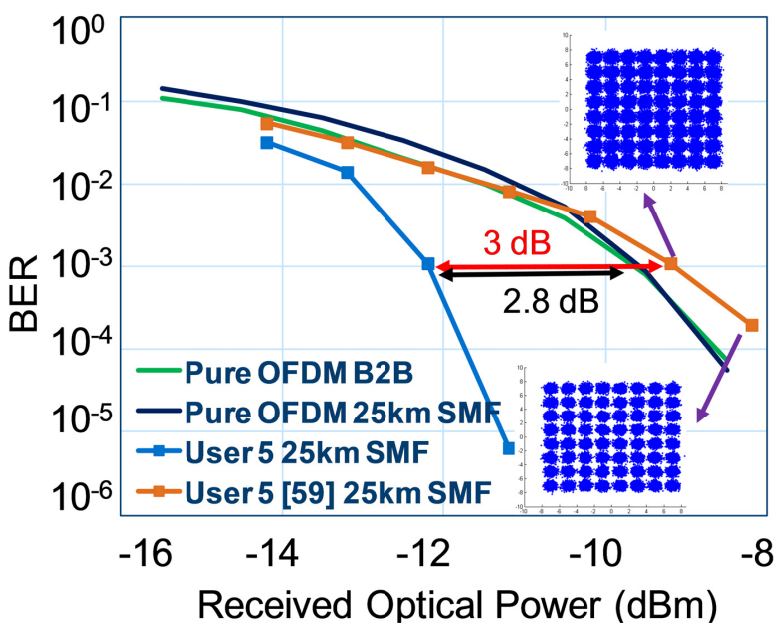

Fig. 5. BER measurements of pure OFDM transmission B2B and after $25 \mathrm{~km}$ of SMF, and 64 QAM OFDM CDMA after $25 \mathrm{~km}$ with single user 5 and user 5 out of two users (Insets: 64 QAM constellation with BER of $1.1 \times 10^{-3}$ and $2 \times 10^{-4}$ ).

More users of 64 QAM however cannot be demonstrated due to the experimental system noise floor consideration that is worse than the FEC limit. Fig. 6 shows the BER curves of 64 QAM OFDM CDMA user 5 decoded in four-user system (code 3, code 5, code 6 and code 7) after $25 \mathrm{~km}$ of SMF and the simulation results under the same noise floor situation. It's found that the BERs measured cannot hit FEC limit and will become stable at around above $1 \times 10^{-3}$. Simulation has been done with fixed noise floor setting with Signal-to-noise-ratio (SNR) of $18 \mathrm{~dB}$ (BER @1 $1 \times 10^{-3}$ ) then converted to optical power as in experiments. The simulation results fit the experiments well. Hence in order to support more users, the power penalty has to be decreased, so in the following experiments the modulation format is then changed to 16 QAM.

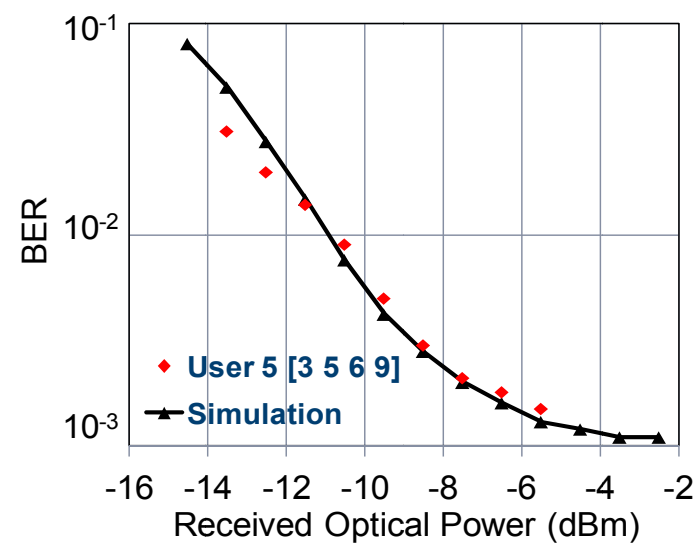

Fig. 6. BER measurements of 64 QAM OFDM CDMA after $25 \mathrm{~km}$ with user 5 out of four users and simulation results with fixed noise floor.
Fig. 7 shows the BER curves for a 16 QAM OFDM-CDMA PON that supports one, two and four users, with an additional $3 \mathrm{~dB}$ splitting loss for each case. However when comparing user 5 and user 9 in a oneuser system, two-user system and four-user system, there are increasing penalties of $1 \mathrm{~dB}, 1.5 \mathrm{~dB}$ and $1.9 \mathrm{~dB}$ at a BER of $1 \times 10^{-3}$ respectively. This is because different spreading codes have different frequency bandwidths and hence suffer from different losses as shown in Fig. 4. As a result, they have different SNRs even in the one-user system. Also as the user number increases, dispersion and crosstalk degrade the channel orthogonality causing a higher power penalty. The spectral efficiency in this system however decreases to $2 \mathrm{bit} / \mathrm{s} / \mathrm{Hz}$ with a single user data rate of $2.5 \mathrm{~Gb} / \mathrm{s}$, but still shows the viability of $16 \mathrm{ONU}$ channels with an aggregate date rate of $40 \mathrm{~Gb} / \mathrm{s}$. This could be increased further if more advanced orthogonal codes, higher order modulation formats or higher code chip rates were used. Of course this also requires a more complex synchronization scheme.

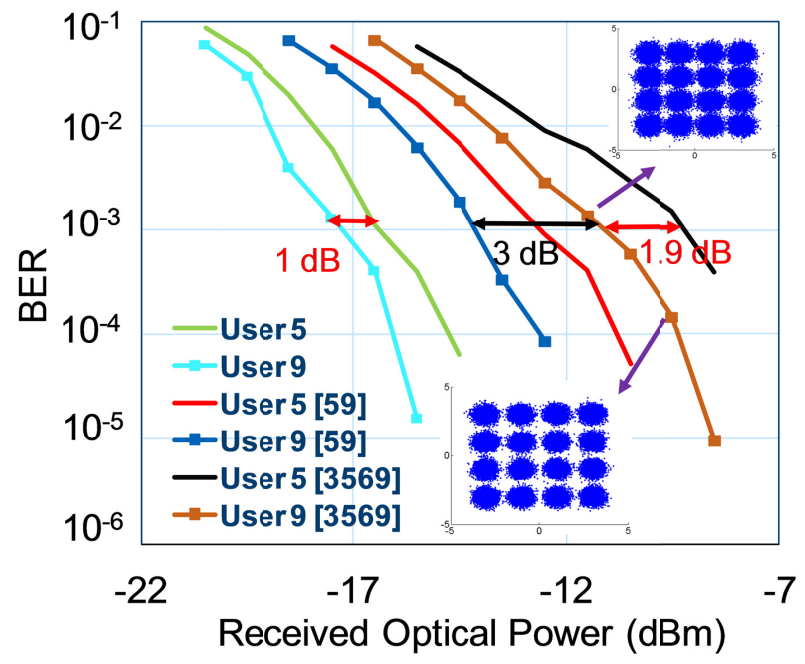

Fig. 7. BER measurements of 16 QAM OFDM CDMA after $25 \mathrm{~km}$ with one, two and four users (Insets: 16 QAM constellations with BER of $1.4 \times 10^{-3}$ and $\left.1.5 \times 10^{-4}\right)$.

We have proposed and demonstrated the feasibility of a novel OFDM-CDMA PON architecture. Two and four users out of 16-user system have been demonstrated with maximum single channel data rate of $3.75 \mathrm{~Gb} / \mathrm{s}$ and potential aggregate data rate $60 \mathrm{~Gb} / \mathrm{s}$. The dispersion penalty is about $0.1 \mathrm{~dB}$ and crosstalk penalty is $0.9 \mathrm{~dB}$ for four users after $25 \mathrm{~km}$ of SMF. The coding gain is $2.8 \mathrm{~dB}$ for $64 \mathrm{QAM}$ OFDM-CDMA single user compared to the case without CDMA, this can be improved further with lower order modulation format. This architecture has the benefits of multiple users' access with bidirectional transmission, coding gain for high power budget margins, spectral efficiency and networking functions with a single wavelength.

We acknowledge Tektronix for supplying arbitrary waveform generator (AWG70001A).

\section{References}

1. Y. Luo, X. Zhou, F. Effenberge, X. Yan, G. Peng, Y. Qian, and Y. Ma, J. Lightw. Technol., 31, 587(2013).

2. N. Cvijetic, J. Lightw. Technol., 30, 384(2012).

3. J. Armstrong, J. Lightw. Technol., 27, 189 (2009).

4. H. Yang, J. Li, B. Lin, Y. Wan, Y. Guo, L. Zhu, L. Li, Y. He and Z. Chen.. J. Lightw. Technol., 31, 2735(2013).

5. X. Hu, L. Zhang, P. Cao, K. Wang and Y. Su. Opt. Express., 20, 8071 (2012).

6. K. Kitayama, Optical Code Division Multiple Access: A Practical Perspetive (Cambridge University Press, 2014).

7. L. Zhang, X. Xin, B. Liu, J. Yu and Q. Zhang. Opt. Express., 18, 18347 (2010).

8. L. Fang, L. Zhou, X. Liu, X. Zhang, M. Sui, F. Effenberger and J. Zhou. Opt. Express., 23, 13499 (2015). 